\title{
A new therapeutic modality involving acridine orange excitation by photon energy used during reduction surgery for rhabdomyosarcomas
}

\author{
TAKAO MATSUBARA ${ }^{1}$, KATSUYUKI KUSUZAKI ${ }^{1,2}$, AKIHIKO MATSUMINE $^{1}$, \\ HIROAKI MURATA ${ }^{3}$, HARUHIKO SATONAKA ${ }^{1}$, KEN SHINTANI ${ }^{1}$, TOMOKI NAKAMURA ${ }^{1}$, \\ HAJIME HOSOI $^{4}$, TOMOKO IEHARA ${ }^{4}$, TORU SUGIMOTO ${ }^{4}$ and ATSUMASA UCHIDA ${ }^{1}$ \\ ${ }^{1}$ Department of Orthopaedic Surgery, Mie Postgraduate School of Medicine, 2-174 Edobashi, Tsu City, \\ Mie Prefecture 514-8507; ${ }^{2}$ Department of Orthopaedic Surgery, Odai Kosei Hospital Sahara 63-8, Odai-cho \\ Taki, Mie Prefecture 519-2404; Departments of ${ }^{3}$ Orthopaedic Surgery and ${ }^{4}$ Pediatrics, Kyoto Prefectural \\ University of Medicine, 465 Kajii-cho Kawaramachi-Hirokoji Kamigyo-ku, Kyoto 602-0841, Japan
}

Received April 30, 2008; Accepted August 20, 2008

DOI: 10.3892/or_00000193

\begin{abstract}
Rhabdomyosarcoma is a common malignant soft tissue that frequently involves bone and major neurovascular structures and resection of deep-seated rhabdomyosarcoma can cause severe dysfunction in the affected limbs. Based on the mouse osteosarcoma model, we developed a new surgical approach involving photodynamic surgery (PDS), photodynamic therapy (PDT) and radiodynamic therapy (RDT) using acridine orange (AO). Six rhabdomyosarcoma cases were treated using this new modality after confirming the effectiveness of AO-PDT on human rhabdomyosarcoma cell lines. All patients had almost normal limb function after surgery, with only one recurrence. Based on these results, AO-PDS, PDT and RDT can be used to preserve excellent limb function in patients with rhabdomyosarcoma involving major nerves and vessels or bones.
\end{abstract}

\section{Introduction}

Rhabdomyosarcoma is the most common malignant soft tissue sarcoma occurring in children and young adults (1). Overall 5 -year survival rates range from 55 to $71 \%$ after surgery with IRS chemotherapy (2-5). Although the effectiveness of chemotherapy in rhabdomyosarcoma is still being investigated, it is clear that local recurrence after surgery is a negative prognostic factor (6). The Intergroup Rhabdomyosarcoma Study Group (IRSG) recommends performing a complete procedure with a wide-margin tumor resection. However,

Correspondence to: Dr Katsuyuki Kusuzaki, Odai Kosei Hospital, Sahara 63-8, Odai-cho Taki, Mie Prefecture 519-2404, Japan

E-mail:k-kusuzaki@okh.miekosei.or.jp

Key words: rhabdomyosarcoma, photodynamic therapy, acridine orange, limb salvage since deep-seated rhabdomyosarcoma arising from the extremities frequently involves or invades nerves, vessels, or bones, wide resection occasionally causes severe dysfunction of the affected limbs. To avoid this scenario, we previously established a new limb salvage strategy involving intralesional or marginal tumor excision supported by photodynamic surgery (PDS), photodynamic therapy (PDT) and radiodynamic therapy (RDT) using acridine orange (AO) in patients with high grade malignant sarcomas (7-10). The present study first confirmed the effectiveness of this approach in rhabdomyosarcoma cell lines and then assessed this approach in patients with rhabdo-myosarcoma of the extremities.

\section{Materials and methods}

In vitro study. Before clinical use, the effect of AO-PDT on human rhabdomyosarcoma cell lines was investigated. Three cell lines, RD TG50 (embryonal), Rh30 (alveolar) and MIERh1, were studied. RD and Rh30 cell lines were purchased from the ATCC (The Global Bioresource Center ${ }^{\mathrm{TM}}$, Manassas, USA) and grown in Dulbecco's RPMI-1640 medium. MIERh1 was established from a human rhabdomyosarcoma of a 17-year-old male in our department. These cells $\left(5 \times 10^{3}\right)$ were cultured in RPMI-1640 containing 10\% fetal bovine serum (FBS) at $37^{\circ} \mathrm{C}$ under a $5 \% \mathrm{CO}_{2}$ atmosphere, using 96-well plates. At $24 \mathrm{~h}$, in pre-confluent cell growth conditions, the medium of the wells was replaced with 0.1 , or $1.0 \mu \mathrm{g} / \mathrm{ml}$ AO (Sigma-Aldrich Chemie Gmbh, Taufkrichen, Germany: Lot No.122K0522) containing RPMI-1640. After $10 \mathrm{~min}$ exposure to AO, 10,000 lx light (containing all wave lengths of visible light) obtained from a $500 \mathrm{~W}$ xenon lamp source (Sanei Electronics Co., Ltd, Osaka, Japan) illuminated the cells to excite the AO bound to the cells. In the control study, the cells were cultured in AO-free or AO-containing medium without subsequent light illumination, or in $\mathrm{AO}$-free medium followed by light illumination. Cell viability in each well was measured using the MTS assay (Promega Corporation, WI, 
USA; Cell titer one solution cell proliferation assay) and the cell viability ratio was calculated based on the cell viability of the studied cells divided by the cell viability of the control cells grown in AO-free medium.

Statistical analysis. The association among the variables was determined by one way ANOVA with Fisher's PLSD post hoc test. P-values $<0.05$ were considered significant.

\section{Clinical study}

Cases. From December 1999 to April 2008, 6 patients (4 males, 2 females; average age, 12 years; range, 11 months to 17 years) with rhabdomyosarcoma were recruited from the Departments of Orthopaedic Surgery of University Hospital of the Kyoto Prefectural University of Medicine and the Mie Postgraduate School of Medicine (Table I). Five patients (cases 1 and 3-6) had primary rhabdomyosarcoma and had never before been treated. One case (case 2) had undergone wide tumor excision for a paratesticular tumor, but subsequently developed femoral bone and lung metastases. This patient had AO-PDT for the femoral bone metastasis to prevent a pathological fracture. On histology, all tumors were alveolar type, high grade malignant rhabdomyosarcoma. Four cases were classified as stage II (cases 1 and 4-6), and two cases were classified as stage IV (cases 2 and 3) based on the IRSG staging classification (11). The follow-up duration ranged from 6 (case 2: died of disease) to 106 months (average, 54 months). All patients received VAC chemotherapy (vincristine, actinomycin-D and cyclophosphamide) according to the Japan Rhabdomyosarcoma Study Group (JRSG) protocol.

Surgical procedure. In all cases, intralesional or marginal tumor excision, which is similar to the conventional macroscopic curettage done for benign soft tissue tumors, was performed (Fig. 1-1). These procedures were used to minimize, to the maximum extent possible, damage to intact muscles and bones, as well as major nerves and vessels that were in close contact or involving the tumor and thereby obtain good limb function after surgery.

For the next step involving AO-PDS, microscopic curettage using an ultrasonic surgical scalpel (Olympus Co. Ltd., Tokyo, Japan) was done in addition to using a fluorescence surgical microscope, under tumor fluorovisualization after local administration of $1 \mu \mathrm{g} / \mathrm{ml}$ of AO solution for $5 \mathrm{~min}$, followed by washing out of the excess $\mathrm{AO}$ solution with saline and excitation with blue light, which most effectively excites AO to emit green fluorescence (Fig. 1, 2-5). The microscope was equipped with an interference filter (450-490 nm) to select the blue light emitted by a xenon lamp and an absorption filter $(>520 \mathrm{~nm})$ to allow for observation of the green AO fluorescence under fluorescence surgical microscope (Carl Zeiss Co., Ltd, Oberkochen, Germany). Microscopic curettage was repeated until the green fluorescence disappeared completely from the remnant tumor mass; AO-PDT was applied to the tumor curettage area by illumination $(100,000 \mathrm{~lx})$ of unfiltered light from the xenon lamp for $10 \mathrm{~min}$, again using the fluorescence surgical microscope. After closure of the surgical wound without washing out the AO solution, AO-RDT was done using the following procedure. In the radiotherapy room, a single session of X-ray irradiation (5 Gy)

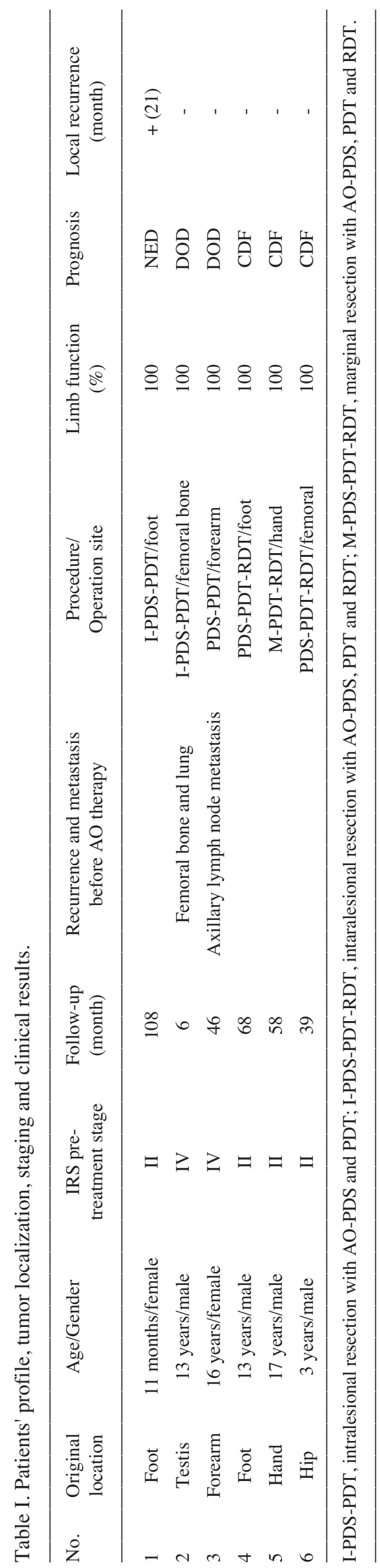




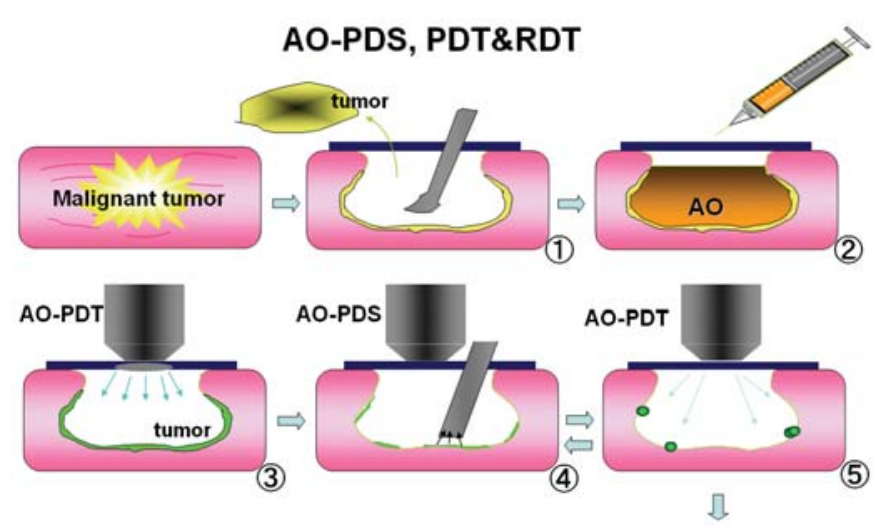

5Gy Radiation (AO-RDT)

Figure 1. AO-PDS, PDT and RDT procedure. (1) Macroscopic intralesional or marginal tumor excision. (2) Local administration of $1 \mu \mathrm{g} / \mathrm{ml}$ of AO solution. (3 and 4) Additional microscopic curettage using an ultrasonic surgical scalpel and a fluorescence surgical microscope under tumor fluorovisualization (AO-PDS). (5) AO-PDT using unfiltered light from the Xenon lamp for $10 \mathrm{~min}$. (6) AO-RDT with a single session of X-ray irradiation (5 Gy).

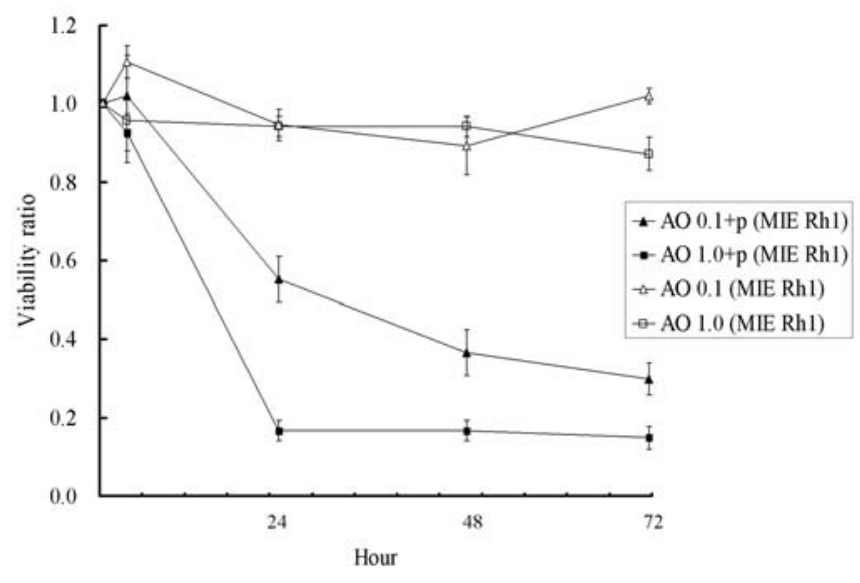

Figure 2. Tumor cell growth inhibition effect of AO-PDT in the human MIERh1 rhabdomyosarcoma cell line. Tumor cells exposed to 0.1 or $1.0 \mu \mathrm{g} / \mathrm{ml}$ AO followed by light illumination die rapidly within $72 \mathrm{~h}$.

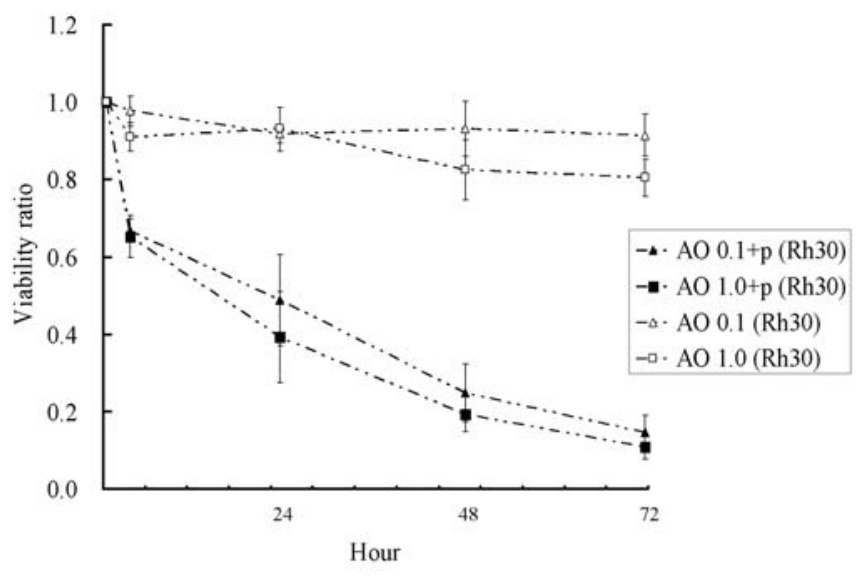

Figure 3. Tumor cell growth inhibition effect of AO-PDT in the human Rh30 rhabdomyosarcoma cell line. Tumor cells exposed to 0.1 or $1.0 \mu \mathrm{g} / \mathrm{ml}$ AO followed by light illumination die rapidly within $72 \mathrm{~h}$.

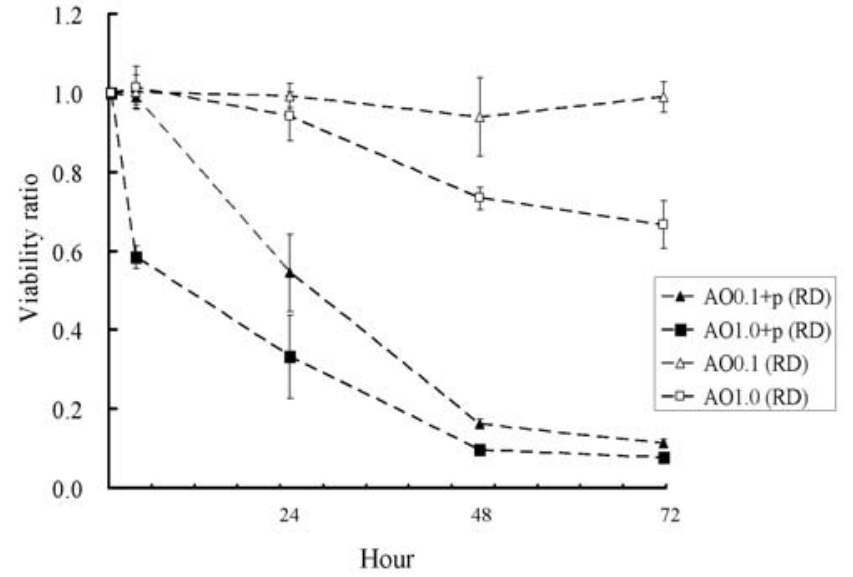

Figure 4. Tumor cell growth inhibition effect of AO-PDT in the human RD50 rhabdomyosarcoma cell line. Tumor cells exposed to 0.1 or $1.0 \mu \mathrm{g} / \mathrm{ml}$ AO followed by light illumination die rapidly within $72 \mathrm{~h}$.

was applied to the resected area immediately in 3 patients (cases 4-6), in order to achieve a strong cytocidal effect of AO excited by low dose X-rays (10). Three patients (cases 1-3) or their family rejected AO-RDT due to complications after radiation. The $\mathrm{AO}$ concentration, light illumination time and dose (lx), and the radiation dose (Gy) were determined based on the data obtained from our basic mouse model studies (7-10). In particular, 5 Gy of X-ray irradiation was found to be sufficient to completely kill mouse osteosarcoma cells exposed to $1 \mu \mathrm{g} / \mathrm{ml} \mathrm{AO}$ within $72 \mathrm{~h}$ (10).

This clinical trial was officially approved by the Ethics Committees of both participating institutions. After a full explanation of the method and purpose of the study was given, each patient and a close family member gave their written informed consent for this clinical study.

Prior to the clinical study, in all cases the sensitivity of each rhabdomyosarcoma to AO was assessed using fresh biopsy specimens, which were exposed ex vivo to $1 \mu \mathrm{g} / \mathrm{ml}$ of $\mathrm{AO}$ solution and then observed using a fluorescence stereoscope after illumination with blue excitation light.

Local tumor recurrence was evaluated by magnetic resonance imaging (MRI) and thallium scintigraphy. Limb function after surgery was evaluated using the Enneking criteria (12). In addition, local and systemic complications induced by AO administration, as well as AO-PDT with or without 5 Gy radiation, were assessed by monitoring clinical symptoms and blood test results.

\section{Results}

In vitro study. The viability ratios of MIERh1 (Fig. 2), Rh30 (Fig. 3) and RD (Fig. 4) cells cultured in AO containing medium at concentrations of 0.1 or $1.0 \mu \mathrm{g} / \mathrm{ml}$ followed by light illumination were significantly decreased at 24,48 and $72 \mathrm{~h}$, compared to the results obtained using control cells cultured in AO-free medium with or without subsequent light illumination, or using cells in various concentrations of AO medium only. Rh30 and RD cells tended to be more sensitive to AO-PDT than MIERh1 cells. These cells died due to rapid cytoplasmic ballooning, similar to that which is observed in mouse osteosarcoma cells after treatment with AO-PDT (7). 


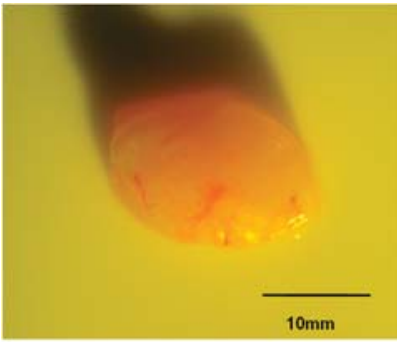

(A)

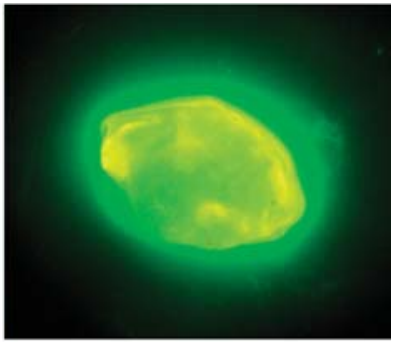

(B)
Figure 5. Fresh tumor biopsy specimen obtained from case 5: (A) rhabdomyosarcoma without AO exposure; (B) rhabdomyosarcoma with $1 \mu \mathrm{g} / \mathrm{ml} \mathrm{AO}$ ex vivo exposure, followed by blue light excitation. Tumor emits green $\mathrm{AO}$ fluorescence indicating sensitivity to $\mathrm{AO}$.

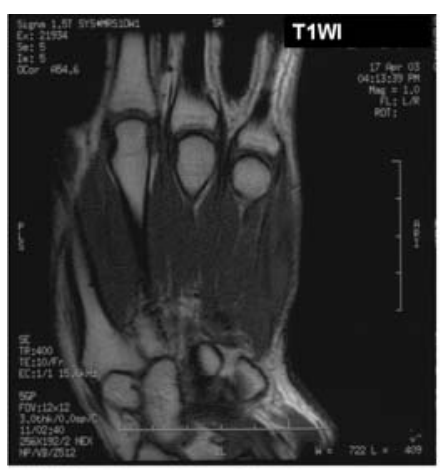

(A)

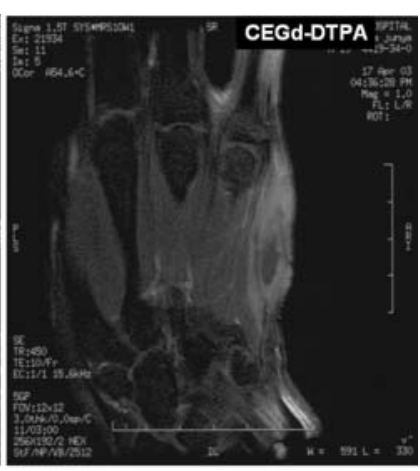

(B)
Figure 6. MRI findings before AO therapy in case 5 with a rhabdomyosarcoma in the hypothenar muscle. (A) Coronal T1-weighted image. (B) Coronal T1-weighted image after Gd-DTPA administration.

Clinical study. All tumor specimens were found to be sensitive to AO, based on their ability to emit green fluorescence after ex vivo exposure to $\mathrm{AO}$ solution and blue-light excitation (Fig. 5).

Two of the patients enrolled in this study died due to lung metastasis (died of disease; DOD), two were continuously disease-free (CDF) and two had no evidence of disease (NED) at the time of writing (April 2008). Limb function for all patients recovered to preoperative levels, a rate of $100 \%$ based on the Enneking criteria (12). None of the patients showed clinical evidence of local or systemic complications that could have been caused by AO administration, AO-PDS, PDT or RDT (Table I).

Case presentation. Case 5 was a 17 -year-old boy who had an alveolar type, high grade, malignant rhabdomyosarcoma arising from the hypothenar muscle of the hand. Gadoliniumenhanced MRI imaging revealed a tumor mass within the hypothenar muscles (Fig. 6). After intensive VAC chemotherapy, the tumor size decreased partially. A wide tumor resection would have sacrificed the hypothenar muscle and the digital nerve. Therefore, to preserve excellent limb function, tumor curettage supported by AO-PDS, PDT and RDT was suggested to the patient and his close family. After obtaining written informed consent, the procedure was performed. On pathology, the tumor had been resected with an

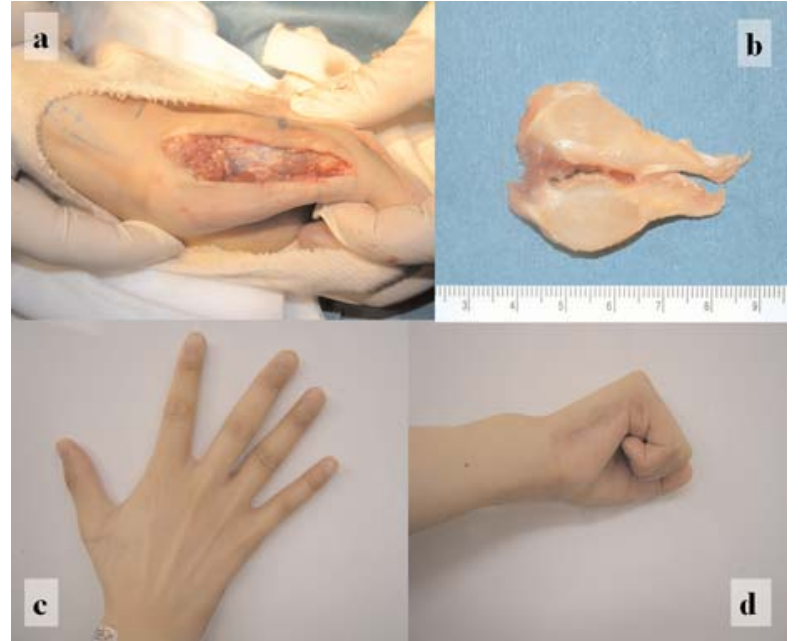

Figure 7. ( $a$ and b) Macroscopic findings of the tumor in the hypothenar muscle during surgery and the cut surface of the resected tumor. (c and d) Finger function after AO therapy.

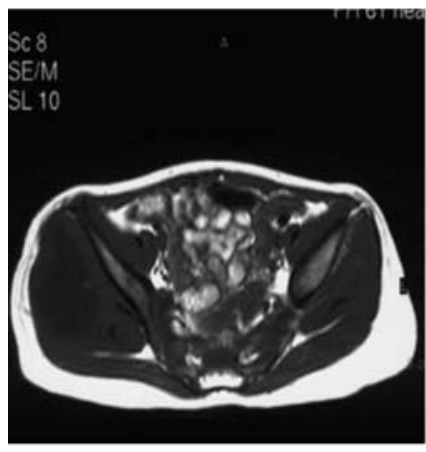

(A)

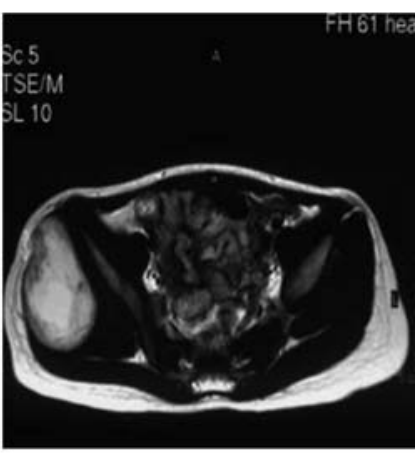

(B)
Figure 8. MRI findings before AO therapy in case 6 with rhabdomyosarcoma of the gluteal muscle. (A) Axial view of T1-weighted image. (B) Axial view of T2-weighted image.

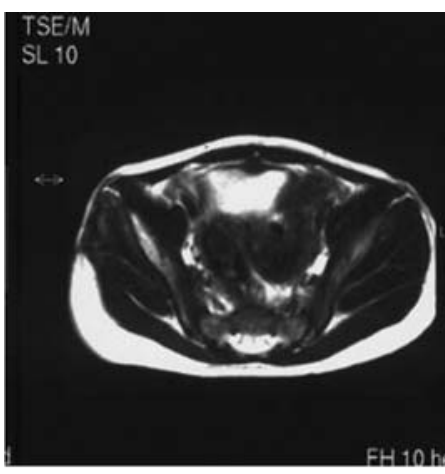

(A)

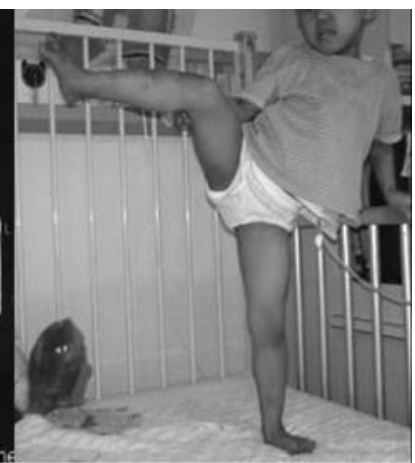

(B)
Figure 9. MRI findings (A) and hip function (B) after AO therapy in case 6 with rhabdomyosarcoma of the right hip. No tumor recurrence is detected in MR images taken 24 months after AO-PDT; hip and lower extremity function is well preserved.

intralesional margin (Fig. 7a and b). Importantly, no tumor recurrence was noted 58 months after surgery and the patient had excellent limb function (Fig. 7c and d). 
Case 6 was a 3-year-old boy who had an alveolar type, high grade, malignant rhabdomyosarcoma arising around the hip. Preoperative MRI revealed that the tumor mass was $>10 \mathrm{~cm}$ in diameter and was localized between the gluteal muscles, in close contact with the sciatic nerve (Fig. 8). To avoid a serious functional deficit of the lower extremity, after preoperative chemotherapy, the AO procedure was performed. On histopathology, many tumor cells were noted at the margin between the tumor and the sciatic nerve; however, no local tumor recurrence was noted 39 months after surgery. The patient had no dysfunction of the lower extremity (Fig. 9).

\section{Discussion}

Previously, we established a new adjuvant approach using acridine orange excited by photon energy for reduction surgery in high grade malignant musculoskeletal tumors $(7-10,13-19)$. This approach consists of three main procedures: photodynamic surgery (PDS), photodynamic therapy (PDT) and radiodynamic therapy (RDT). During PDS, after the tumor is excised with an intralesional margin or a marginal margin with minimal damage to normal tissues, especially major nerves and vessels, a dilute AO solution is applied to the resected tumor area to expose the residual tumor mass to AO. Using a xenon lamp and a fluorescence surgical microscope equipped with an interference filter, blue light excitation is selected. Under these conditions, residual tumor mass emits green fluorescence due to the binding of AO to RNA, DNA and lysosomes or acidic vesicles present in tumor cells $(13,14)$. This green fluorescence is readily visible using the fluorescence microscope equipped with an absorption filter. Since AO is retained much longer in malignant tumor cells than in normal cells, the green fluorescence is seen only in the tumor mass. Therefore, it is possible to distinguish residual tumor mass from normal tissue. Using an ultrasonic surgical knife, tumor emitting green fluorescence can be resected. The PDS procedure can be repeated until no tumor fluorescence is noted. Next, photodynamic therapy (PDT) is applied to the area where surgery was done using unfiltered light from the xenon lamp for $10 \mathrm{~min}$. AO excited by photon energy produces activated oxygen through a type II photoreaction and activated oxygen oxidizes the cell membrane and the lysosomal membrane. This results in rapid death of most tumor cells due to cell swelling and rupture (necrosis). Tumor cells that survive initially subsequently die within $72 \mathrm{~h}$ due to apoptosis caused by DNA, RNA or mitochondrial damage. Therefore, the PDT procedure can kill residual tumor cells that survive following PDS. However, it is difficult to kill tumor cells that are covered or sealed with muscles. Therefore, in the final RDT procedure, after the surgical wound is closed without washing of the AO solution, the surgical area is immediately irradiated with 5 Gy of X-rays in the radiotherapy room. The AO excited by X-ray energy can also kill tumor cells through activated oxygen, since X-rays have much stronger photon energy than visible light. X-ray irradiation has the advantage of reaching deeper areas of the human body than a light beam, even though it is more injurious to normal tissues. Therefore, with the RDT procedure, tumor cells sealed within muscles can be killed. One session of such low dose X-rays does not carry the risk of the development of radiation complications, such as burning, skin fibrosis, or secondary malignancy.

In the present rhabdomyosarcoma study, the in vitro study results using 3 different human rhabdomyosarcoma cell lines showed that the cells were sensitive to AO and that AO-PDT was effective in killing these cells; these results are similar to those we previously reported with respect to mouse osteosarcoma cells (7-10) and human synovial sarcoma cell lines (18). In the clinical part of the study, since fresh biopsy materials obtained from the cases emitted green fluorescence from $\mathrm{AO}$ after ex vivo $\mathrm{AO}$ exposure followed by blue light illumination, it was confirmed that these tumors were sensitive to AO. Treatment with AO-PDS and PDT was followed by AO-RDT in 3 cases and not followed by AO-RDT in 3 cases; only one case developed local recurrence. This particular case was treated with AO-PDS and PDT without RDT after intralesional tumor excision (curettage) because her parents did not agree to radiotherapy due to the risk of secondary malignancy; local recurrence appeared 21 months after treatment. It has been noted that rhabdomyosarcomas treated only with curettage without intensive radiotherapy can recur within 3 months. Therefore, even in this case, AO-PDS and PDT appear to have been effective for local control. Of note, this patient is still alive after a below knee amputation for recurrent tumor. No recurrences were noted in cases treated with AO-PDS, PDT and RDT.

Local control is one of the most important prognostic factors in rhabdomyosarcomas. Therefore, the IRSG recommends wide resection or amputation for rhabdomyosarcomas arising in the extremities. However, after such resections, patients have serious limb function deficits. Even though it is understood that life is much more important than limb function, young patients are left with a serious, long-term, physical and mental handicap after the disease has been cured. AO-PDS, PDT and RDT appear to be helpful to avoid such handicaps.

Based on the experimental and clinical outcomes described, it appears that the use of AO-PDS, PDT and RDT is useful for local control after reduction surgery in rhabdomyosarcoma patients. Given the encouraging results of this preliminary study, further studies involving larger numbers of patients and longer follow-up periods are needed.

\section{References}

1. Weiss SW and Goldblum JR: Enzinger and Weiss's Soft Tissue Tumors. Fourth edition, Mosby, St. Louis, 2001

2. Breneman JC, Lyden E, Pappo AS, Link MP, Anderson JR, Parham DM, Qualman SJ, Wharam MD, Donaldson SS, Maurer HM, Meyer WH, Baker KS, Paidas CN and Crist WM: Prognostic factors and clinical outcomes in children and adolescents with metastatic rhabdomyosarcoma a report from the Intergroup Rhabdomyosarcoma Study IV. J Clin Oncol 21: 78-84, 2003.

3. Maurer HM, Beltangady M, Gehan EA, Crist WM, Hammond D, Hays DM, Heyn R, Lawrence W, Newton W and Ortega J: The Intergroup Rhabdomyosarcoma Study-I. A final report. Cancer 61: 209-220, 1988.

4. Crist WM, Gehan EA, Ragab AH, Dickman PS, Donaldson SS, Fryer C, Hammond D, Hays DM, Herrmann J and Heyn R: The Third Intergroup Rhabdomyosarcoma Study. J Clin Oncol 13: 610-630, 1995 
5. Raney RB, Anderson JR, Barr FG, Donaldson SS, Pappo AS, Qualman SJ, Wiener ES, Maurer HM and Crist WM: Rhabdomyosarcoma and undifferentiated sarcoma in the first two decades of life: a selective review of intergroup rhabdomyosarcoma study group experience and rationale for Intergroup Rhabdomyosarcoma Study V. J Pediatr Hematol Oncol 23: 215-220, 2001.

6. Mameghan H, Fisher R, Tobias V, Kern IB, O'Gorman-Hughes D, Vowels $\mathrm{M}$ and Mameghan J: Local failure in childhood rhabdomyosarcoma and undifferentiated sarcoma: prognostic factors and implications for curative therapy. Med Pediatr Oncol 21: 88-95, 1993.

7. Kusuzaki K, Minami G, Takeshita H, Murata H, Hashiguchi S, Nozaki T, Ashihara T and Hirasawa Y: Photodynamic inactivation with acridine orange on a multidrug-resistant mouse osteosarcoma cell line. Jpn J Cancer Res 91: 439-445, 2000.

8. Kusuzaki K, Aomori K, Suginoshita T, Minami G, Takeshita H, Murata H, Hashiguchi S, Ashihara T and Hirasawa Y: Total tumor cell elimination with minimum damage to normal tissues in musculoskeletal sarcomas by photodynamic reaction with acridine orange. Oncology 59: 174-180, 2000.

9. Kusuzaki K, Suginoshita T, Minami G, Aomori K, Takeshita H, Murata H, Hashiguchi S, Ashihara T and Hirasawa Y: Fluorovisualization effect of acridine orange on mouse osteosarcoma. Anticancer Res 20: 3019-3024, 2000.

10. Hashiguchi S, Kusuzaki K, Murata H, Takeshita H, Hashiba M, Nishimura T, Ashihara T and Hirasawa Y: Acridine orange excited by low-dose radiation has a strong cytocidal effect on mouse osteosarcoma. Oncology 62: 85-93, 2000.

11. Qualman SJ, Coffin CM, Newton WA, Hojo H, Triche TJ, Parham DM and Crist WM: Intergroup Rhabdomyosarcoma Study: update for pathologists. Pediatr Dev Pathol 6: 550-561, 1998.

12. Enneking WF, Dunham W, Gebhardt MC, Malawar M and Prichard DJ: A system for the functional evaluation of reconstructive procedures after surgical treatment of tumors of the musculoskeletal system. Clin Orthop 286: 241-246, 1993.
13. Kusuzaki K, Murata H, Takeshita H, Hashiguchi S, Nozaki T, Emoto K, Ashihara T and Hirasawa Y: Intracellular binding sites of acridine orange in living osteosarcoma cells. Anticancer Res 20: 971-976, 2000.

14. Matsubara T, Kusuzaki K, Matsumine A, Shintani K, Satonaka H and Uchida A: Acridine orange used for photodynamic therapy accumulates in malignant musculoskeletal tumors depending on pH gradient. Anticancer Res 26: 187-193, 2006.

15. Satonaka H, Kusuzaki K, Matsubara T, Shintani K, Wakabayashi T, Matsumine A and Uchida A: Extracorporeal photodynamic image detection of mouse osteosarcoma in soft tissues utilizing fluorovisualization effect of acridine orange. Oncology 70: 465-473, 2006.

16. Kusuzaki K, Murata H, Matsubara T, Satonaka H, Wakabayashi T, Matsumine A and Uchida A: Acridine orange could be an innovative anticancer agent under photon energy. In Vivo 21: 205-214, 2007.

17. Kusuzaki K, Murata H, Matsubara T, Miyazaki S, Okamura A, Seto M, Matsumine A, Hosoi H, Sugimoto T and Uchida A: Clinical trial of photodynamic therapy using acridine orange with/without low dose radiation as new limb salvage modality in musculoskeletal sarcomas. Anticancer Res 25: 1225-1235, 2005.

18. Kusuzaki K, Murata H, Matsubara T, Miyazaki S, Shintani K, Seto M, Matsumine A, Hosoi H, Sugimoto T and Uchida A: Clinical outcome of a novel photodynamic therapy technique using acridine orange for synovial sarcomas. Photochem Photobiol 81: 705-709, 2005.

19. Yoshida K, Kusuzaki K, Matsubara T, Matsumine A, Kumamoto T, Komada Y, Naka N and Uchida A: Periosteal Ewing's sarcoma treated by photodynamic therapy with acridine orange. Oncol Rep 13: 279-282, 2005. 